(Aus dem thierphysiologischen Laboratorium der landwirthschaftlichen Hochschule zu Berlin.)

\title{
Beiträge zur Kenntniss der Verdaulichkeit der Milch und des Brodes.
}

\author{
Von
}

\section{Dr. A. Magnus-Levy.}

In einem vor $1 \frac{1}{2}$ Jahren gemeinschaftlich mit Prof. $\mathrm{Zuntz}$ veröffentlichten Ausnützungsversuch hatte Verfasser Gelegenheit gehabt, an einem einzelnen Fall die Richtigkeit von Bunges bekannter Behauptung zu prüfen, derzufolge der regelmässige Genuss von Alcohol die Leistungsfähigkeit des Verdauungsapparates gegenüber einfacher Pflanzenkost herabsetzen solle. B u n g e (1) hatte diesen Satz u. a. anlässlich einer Besprechung der Ausnutzungsversuche der Münchener physiologischen Schule angeführt und deren Resultate als theilweise ungünstig und als nicht verallgemeinerungsberechtigt hingestellt. Nun ist es sicher begründet, vor der Verallgemeinerung gewisser Zahlen; die nur an wenig Individuen in einzelnen Versuchen von e in e $m$ noch so verdienstvollen Beobachter gewonnen sind, zu warnen, und $\mathrm{Zuntz}$ und Mag n u s-Lev y (2) haben in der That gesehen, dass sie selbst das Eiweiss des Brodes weit besser ausnützten, als es in den Münchener Versuchen der Fall gewesen. Konnten sie somit zwar individuelle Unterschiede deutlich nachweisen, so war eine schlechtere Ausnutzung des Eiweisses durch Biergenuss in ibrem Versuch (L. trank 1,81 Bier täglich, Z. enthielt sich vollständig geistiger (Getränke) nicht zu bemerken. Eine weitere Prüfung dieser Frage erschien immerhin von Interesse, und so ging Verfasser gern auf den Vorschlag des Herrn Prof. Z $\mathfrak{u} \mathbf{t z}$ ein, einige Versuche an dessen Sohn L. Z. vorzunehmen. Derselbe damals (März 1891) 16 Jahre alt, Gymnasiast, gut entwickelt, wog 57,5 Kilo, hatte, dank der Erziehung des Vaters, kaum je Alcohol genossen, oder das Verlangen nach solchem versptirt, und war andererseits an reich- 
Beiträge zur Kenntniss der Verdaulichkeit der Milch und des Brodes. $\mathbf{5 4 5}$

lichen Milchgenuss gewöhnt. Ein solches Individuum schien besonders geeignet, $\mathrm{um}$ die von $\mathrm{Bung}$ e behauptete bessere Verwerthung der Nahrungsmittel bei Abwesenheit von'Alcohol zu studiren.

Zwei kleinere Versuchsreihen sollten gemacht werden, eine 3 tägige mit ausschliesslicher Milchdiät, da L. Z. Milch stets reichlich genoss und gut vertrug, was bei wenig Erwachsenen der Fall ist. Daran sollte ein Versuch sich schliessen mit Milch, Brod und Butter im Hinblick speciell auf die Verwerthung des Brodes, da Zuntz und M. L., wie schon oben erwähnt, das Brod besser ausgenutzt batten, wie frühere Forscher, und also auch hier, wie für den Milchversuch ein grösseres Vergleichsmaterial vorlag. Verfasser wollte, wie in den friberen mit Prof. $Z u n t z$ gemeinschaftlich angestellten Reihen, auch diesmal Parallelversuche am ejgenen Körper mit den gleichen Nahrungsmitteln unter Zugabe von Alcohol (Cognac) machen, musste aber leider in der Mitte des zweiten Tages mit dem Milchgenuss aufhören, der bei ihm ausser Durchfällen die auch anderen Untersuchern (Rubner, Hoffmann, Sas setzky, Herfeldt, Müller) bekannte nervöse Depression hervorrief und zur Aufgabe des Experimentes zwang. Dagegen vertrug L. Z. sowohl die ausschliessliche Milchdiät, wie die Zusammenstellung von Milch, Brod and Butter, bis auf leichte Obstipationsbeschwerden während des ersten Versuches ausgezeichnet.

1. A ussebliessliebe Milchdiät.

In den ersten 3 Tagen lebte $Z$. aussehliesslich von (sterilisirter) Milch, die er mit Behagen bei bestem Wohlbefinden in freigestellter Menge genoss. Er führte ein ${ }^{1}$ ):

1) Analyse der Nahrungsmittel:

\begin{tabular}{|c|c|c|c|c|c|c|c|}
\hline & $\begin{array}{l}\text { Stick- } \\
\text { stoff }\end{array}$ & $\begin{array}{c}\text { Ei- } \\
\text { weiss }\end{array}$ & Fett & $\begin{array}{l}\text { Kohlenhydrate als } \\
\text { Stärke berechnet }\end{array}$ & Asche & F.R. & $\mathrm{H}_{2} \mathrm{O}$ \\
\hline Milch & 0,469 & 2,96 & 3,11 & $4,62 \mathrm{MZ}=4,38 \mathrm{St}$ & 0,67 & 11,33 & 88,67 \\
\hline Weissbrod & 1,177 & 7,36 & 0,07 & $55,59 n$ & 0,77 & 63,79 & 36,21 \\
\hline Butter & 0,117 & 0,73 & 81,77 & - & 1,81 & 84,30 & 15,70 \\
\hline Rohrzucker & - & - & $\longrightarrow$ & $100 \% \mathrm{R} Z=95,00 "$ & - & $|100,00|$ & 0,00 \\
\hline
\end{tabular}

Sämmtl. Analysen sind das Mittel aus mehreren gut stimmenden EinzelAnalysen verschiedener Proben.

Die Bolle'sche sterilisirte Milch war in den verschiedenen Flaschen von 


\begin{tabular}{l|c|c|c|c|c|c|c}
\hline Datum & $\begin{array}{c}\text { Milch- } \\
\text { menge } \\
\text { in gr }\end{array}$ & N 1) & Eiweiss & Fett & $\begin{array}{c}\text { K. h. } \\
\text { als Stärke }\end{array}$ & Asche & Tr. S. \\
\hline 23. III. & 3090 & 14,48 & 91,5 & 96,1 & 135,3 & 20,70 & \\
24. III. & 3650 & 17,12 & 108,0 & 113,5 & 159,9 & 24,46 & \\
25. III. & 3764 & 17,65 & 111,4 & 117,1 & 164,9 & 25,22 & \\
\hline Summe & 10504 & 49,25 & 310,9 & 326,7 & 460,1 & 70,38 & 1190,2 \\
pro Tag & 3,501 & 16,42 & 103,6 & 108,9 & 153,4 & 23,46 & 396,7
\end{tabular}

Die Versuchstage dauerten von $7 \frac{1}{2}$ Uhr frih bis $71 / 2$ Uhr des anderen Tages. Der Koth dieser und der nächsten Reihe konnte dureh Fleiseh und Preissel- resp. Heidelbeeren gut von dem bei gemischter Kost abgegrenzt, der Milchkoth von dem Brod-Milchkoth durcb Kohlenpulver leicht unterschieden werden. Die Kothentleerung war schmerzhaft und verzögert, der erste und letzte Koth erschienen je 48 Stunden nach Beginn resp. Ende des Versuches. Die maiskolbenähnlichen, kleinen, harten, in der Porzellanschale klappernden Knollen zerfielen beim Trocknen zu einem leichten, eigenthümlich braunroth gefärbten Pulver (so auch bei Rubner).

Obigen Einnahmen stellen sich folgende Ausgaben im Koth ${ }^{2}$ ) gegenuiber:

gleicher Mischung. Sämmtliche Stoffe in ihr, direct einzeln bestimmt, ergaben $11,33 \%$ feste Bestandtheile, die direkte Wasserbestimmung dagegen 11,13\% F. R. Das Eiweiss direct bestimmt $=2,96 \%$, berechnet zu $2,93 \%$.

Im Brod und Butter ist das Eiweiss = dem 6,25\% Betrag des Stickstoffs gesetzt; die Stärke des Brodes aus der Differenz berechnet, der Milchzucker der Butter vernachlässigt, der Robrzucker zu 100\% gerechnet; Rohrund Milchzucker sind nach dem Verhältniss der Moleculargewichte auf Stärke umgerechnet.

1) Sämmtl. Angaben in Grammen.

2) Der trockene Milchkoth enthielt

$$
\text { in } \begin{aligned}
65,56 \mathrm{gr}: 45,01 \text { organ. Substanz } & =68,66 \%, \\
20,55 \text { Asche } & =31,34 \%, \\
2,515 \mathrm{~N} & =3,84 \%, \\
14,82{ }^{\text {"Fett }} \text { " } & =22,61 \%, \\
0,00 \text { Kohlenhydrate } & =0,00 \% .
\end{aligned}
$$


Beiträge zur Kenntniss der Verdaulichkeit der Milch und des Brodes. 547

\begin{tabular}{|c|c|c|c|c|c|c|}
\hline & $\mathrm{N}$ & "Fett" & "Stärke" & Asche & Tr. S. & org. Tr.S. \\
\hline Aufnahme & 49,25 & 326,7 & 460,1 & 70,38 & 1190,2 & 1120,0 \\
\hline A usgabe & 2,52 & 14,8 & 0,0 & 20,55 & 65,6 & 45,0 \\
\hline Resorb. in $\%$ & 94,9 & 95,5 & 100,0 & 70,8 & 94,5 & 96,0 \\
\hline Verlust in $\%$ & 5,1 & 4,5 & 0,0 & 29,2 & 5,5 & 4,0 \\
\hline
\end{tabular}

Die Stickstoff bilanz dieser Tage war negativ trotz der grossen Menge des zugeführten Eiweisses (104 gr), da die Nahrung sicher ungenïgend war, wie dies auch das stetige Absinken des Körpergewichtes von 58,3 auf $57,55,57,14,57,01$ Kilo anzeigte.

Stickstoff-Bilanz.

\begin{tabular}{|c|c|c|c|c|c|}
\hline & \multirow{2}{*}{ Einnahme } & \multicolumn{2}{|c|}{ Ausgabe im } & \multirow[b]{2}{*}{ Summa } & \multirow{2}{*}{$\begin{array}{c}\text { Verlust } \\
\text { vom Körper }\end{array}$} \\
\hline & & Urin & Koth & & \\
\hline 1. & 14,48 & 16,68 & 0,84 & 17,52 & 3,04 \\
\hline 2. & 17,12 & 19,48 & 0,84 & 20,32 & 3,20 \\
\hline 3. & 17,65 & 19,94 & 0,84 & 20,78 & 3,13 \\
\hline Sa. & 49,25 & 56,10 & 2,52 & 58,62 & 9,37 \\
\hline
\end{tabular}

Auf Grund ähnlicher Berechnungen, wie sie seinerzeit von Prof. Zuntz und dem Verfasser ${ }^{1}$ ) auf Grund der Rubner'schen Zahlen ausgeführt sind, stellt sich der Verbrennungswerth des im Tage resorbirten Nährmaterials folgendermaassen:

$98,4 \mathrm{gr}$ Eiweiss brauchen $131,5 \mathrm{gr} 0_{2}$, liefern $141,4 \mathrm{gr} \mathrm{CO}_{2}$, 398,6 Calorien, $104,0 \mathrm{gr}$ Fett " 297,5 , " \$ 289,4 》 956,0 ,

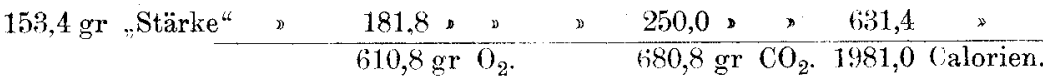

Die Verbrennungswärme des Caseins ist etwas grösser als die des Muskelfleisehs, für die Rub ne $\mathrm{r}$ seine Berechnungen angestellt hat. Setzen wir dementsprechend statt 1981 ca. $2020 \mathrm{Ca}^{-}$ lorien, so kommen bei 57,5 Kilo Körpergewicht 35 Calorien auf das Kilo, welche Wärmemenge sicher nicht ausreichte, den Wärme-

1) Zuntzu. Magnus-Levy, Pfïger's Archiv, Bd 49, S. 445.

$1 \mathrm{gr}$ Eiweiss verbraucht $1,396 \mathrm{gr} \mathrm{O}$, liefert $1,437 \mathrm{gr} \mathrm{CO}_{2}$ und 4,000 Calorien,
1. Butterfett
1. Stärke
$2,861 \times$
2,783 ,
- 9,192
1,185
$1,630>, 4,116$, 
verlust und die Arbeitsleistung eines jungen lebhaften, nicht ganz ausgewachsenen Menschen zu decken. Respirationsversuche an dem jungen Manne hatten ergeben, dass bei vollkommener Ruhe und Hunger in 24 Stunden fast $500 \mathrm{gr} \mathrm{O}_{2}$ verbraucht worden wären. Für die Verdaung der Milch und die Bewegung wären weit mehr als $110 \mathrm{gr} \mathrm{O}_{2}$ verbraucht worden, $d$. h. es musste nach der Verbrennung der zugeführten Nahrung Material vom Körper oxydirt werden; und zwar wurde, wie aus obiger Stickstoffbilanz hervorgeht, neben Fett noch Eiweiss abgegeben. Letzteres war auch in den Versuchen von Prausnitz (3), Hoffmann (4) und Herfeldt (5) der Fall; dagegen konnten sich sämmtliche 5 Kinder von Camerer (6) bei ausschliesslicher Milchdiät annähernd auf dem Stichstoffgleichgewicht halten, obgleich nur bei den 3 jüngsten das Nahrungsbedürfniss gedeckt war. Stickstoff- und Körpergleichgewicht bestand auch in fast allen Versuchen von Slatkowsky (7), bei denen grosse, das Nahrungsbedürfniss deckende und tiberschreitende Milchmengen (bis zu 5,2 Liter) gegeben wurden. Höchst auffallender Weise befand sich auch Rubner's (8) Versuchsmensch von 71 Kilo Gewicht bei circa 2,45 Liter Milch pro die 3 Tage lang im Stickstoffgleichgewicht ${ }^{1}$ ).

\section{Ernährung mit Milch, Brod und Butter.}

Unmittelbar an diesen Versuch schloss sich ein zweiter an, in dem Z. neben Mileh noch Weissbrod (eigens für diesen Zweck im Hause gebacken) und Butter genoss. Er verzehrte pro Tag gleichmässig $2177 \mathrm{gr}$ Milch, $600 \mathrm{gr}$ Brod, $88 \mathrm{gr}$ Butter; 28,5 gr Rohrzucker $=27 \mathrm{gr}$ Stärke am ersten Tage genossen ${ }^{2}$ ), ergeben anf 3 Tage vertheilt je 9 gr Stärke.

1) Wahrscheinlich hat auch Rubner's Versuchsmensch, Dr. G., ein Stickstoffdeficit gehabt. R. berechnet den (nicht analysirten) Stickstoffgehalt der Milch nach Voit zu $0,64 \%$. Praus nitz fand ihn in einem ähnlichen Versuch in München zu nur 0,43. Die Voi t'sche Procentzahl für das Eiweiss der Milch 4,1 steht weit über der Mittelzahl von König $(3,4 \%)$. Möglicherweise ist die Milch stickstoffärmer gewesen als $\mathrm{B}$ u bn e r annimmt.

2) Als Zuckerwasser, um abfïhrend zu wirken. 
Beiträge zur Kenntniss der Verdaulichkeit der Milch und des Brodes. 549

Einn a h m e :

\begin{tabular}{|c|c|c|c|c|c|c|}
\hline & $\mathrm{N}$ & Eiweiss & Fett & „Stärke & Asche & Tr. Sbst. \\
\hline $2177 \mathrm{gr}$ Milch & 10,21 & 64,4 & 67,7 & 95,4 & 14,59 & 246,7 \\
\hline $600 \mathrm{gr}$ Brod & 7,06 & 44,2 & 0,4 & 333,5 & 4,62 & 382,7 \\
\hline $88 \mathrm{gr}$ Butter & 0,10 & 0,6 & 72,0 & - & 1,59 & 74,2 \\
\hline 9,5 gr Rohrzucker & - & - & - & 9,0 & - & 9,5 \\
\hline Pro Tag & 17,37 & 109,2 & 140,1 & 437,9 & 20,80 & 713,1 \\
\hline In 3 Tagen & 52,11 & 327,6 & $4 \subset 0,3$ & 1313,7 & 62,40 & 2139,3 \\
\hline
\end{tabular}

Der Koth, dessen Abgrenzung oben angegeben ist, war durchgängig dunkler als der Milchkoth und weniger consistent; nur gelegentlich fanden sich Andeutungen von „Maiskolben“; auch diesmal folgten die Entleerungen der Nahrungsaufnahme um 48 Éviuten nach.

Den Einnahmen der ganzen Reihe stellen sich im Koth ${ }^{1}$ ) folgende Ausgaben genïber:

\begin{tabular}{l|c|c|c|c|c|c}
\hline & $\mathrm{N}$ & „Fett" & „Stärke & Asche & . Tr. S. & Organ. S. \\
\hline Einnahme & 52,11 & 420,3 & 1313,7 & 62,40 & 2139,3 & 2067,0 \\
Ausgabe & 4,46 & 27,9 & Spuren & 18,17 & 96,75 & 78,6 \\
\hline Resorbirt in \% & $91,5 \%$ & $93,3 \%$ & $100,0 \%$ & $70,9 \%$ & $95,5 \%$ & $96,1 \%$ \\
Verlust in \% & 8,5, & 6,7, & 0,0, & 29,1, & 4,5, & 3,9,
\end{tabular}

Berechnet man den auf die einzelnen Nahrungsstoffe (Milch, Brod, Butter) fallenden Verlust unter der Annahme, dass die Milch grenau wie im ersten Versuche ausgenutzt worden sei, so kommt man zu folgendem Resultat:

1) Der Brodmilchkoth $=447,40$ frisch $=106,7 \mathrm{gr}$ lufttr. $=96,75 \mathrm{gr}$ trocken

$$
\begin{aligned}
\text { mit } 78,58 \text { organ. Subst. } & =81,22 \%, \\
\Rightarrow 18,17 \text { Asche } & =18,78 \% \\
\Rightarrow \quad 4,459 \mathrm{~N} & =4,61 \% \\
" 27,90 \text { "Fett" } & =28,84, \\
\text { " Spuren "Stärke" } & =0,50 "
\end{aligned}
$$


Aufnahme in 3 Tagen.

\begin{tabular}{r|r|r|r|r|r}
\hline & $\mathrm{N}$ & "Fett $^{\natural ;}$ & Asche & Tr. S. & Organ. S. \\
\hline $6531 \mathrm{gr}$ Milch & 30,63 & 20,31 & 43,77 & 740,0 & 696,2 \\
$1800 \mathrm{gr}$ Brod & 21,18 & 1,2 & 13,86 & 1148,2 & 1134,3 \\
$264 \mathrm{gr}$ Butter & 0,30 & 216,0 & 4,77 & 222,6 & 217,8 \\
$28,5 \mathrm{gr}$ Rohrzucker & - & - & - & 28,5 & 28,5 \\
\hline Summa & 52,11 & 420,3 & 62,40 & 2139,3 & 2076,8
\end{tabular}

Von der Mileh erscheinen bei Verlusten von $5,1 \% \mathrm{~N}, 4,5 \%$ Fett, 29,2\% Asche, 5,5\% Tr.S und 4\% organ. Substanz folgende Mengen im Koth wieder:

\begin{tabular}{|c|c|c|c|c|c|}
\hline & $\mathrm{N}$ & „Fett" & A sche & Tr. S. & Organ. S. \\
\hline Von der Milch . . . . & 1,56 & 9,1 & 12,78 & 40,7 & 27,9 \\
\hline Im Koth finden sich . . . & 4,46 & 27,9 & 18,17 & 96,75 & 78,6 \\
\hline $\begin{array}{l}\text { Differenz } \cdot \cdot \cdot \cdot \cdot \cdot \cdot \\
\text { Aufgenommen ist : }\end{array}$ & 2,90 & 18,8 & 5,39 & 56,05 & 50,7 \\
\hline In Brod gr $\mathbf{N} \ldots \ldots$ & 21,18 & - & - & - & - \\
\hline In der Butter gr Fett . . & - & 216,0 & - & - & - \\
\hline In Brod und Butter Asche. & - & - & 18,63 & - & - \\
\hline In Brod, Butter, Zucker Tr.S. & $一$ & - & - & 1399 & - \\
\hline$" \quad " \quad " \quad$ Org.s. & - & - & - & - & 1380 \\
\hline Mithin Vexlust in $\%$. . & 13,7 & 8,7 & 28,9 & 4,0 & $.3,7$ \\
\hline
\end{tabular}

Bei dem geringen Stickstoffgehalt der Butter, und der verschwindenden Menge Fett im Brod kann der Stickstoffverlust im Koth mit Recht nur auf das Brod, der Fettverlust auf die Butter bezogen werden.

Es lieferten pro Tag die resorbirten Nährstoffe an $\mathrm{CO}_{2}, \mathrm{Ca}-$ Iorien, resp. verbrauchten an Sauerstoff folgende Mengen:

$99,9 \mathrm{gr}$ Eiweiss brauchten $133,5 \mathrm{gr} \mathrm{O}_{2}$, liefern 143,6 $\mathrm{gr} \quad \mathrm{CO}_{2}, 399,6$ Calorien,

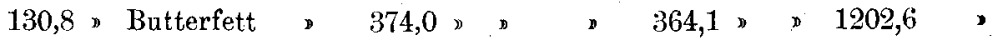

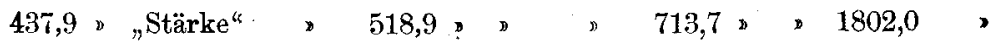
1026,4 gr O $\mathrm{O}_{2}, \quad 1221,4 \mathrm{gr} \mathrm{CO}, 3404,2$ Calorien, Dazu ein Plụs von Calorien für das Casein (s. oben). . + 24 
Beiträge zur Kenntniss der Verdaulichkeit der Milch und des Brodes. 551

i. e. bei 57,6 mittlerem Körpergewicht 59,5 Calorien pro Körperkilo. Bei dieser das Bedturfniss weit ihersteigenden Menge von Nährstoffen nahm der Körper an Gewicht zu von 57,01 auf 57,62 , 58,21 und 58,07 , ohne indessen den Stickstoffverlust der vorigen Tage wieder einzubringen.

Am ersten Tage gab Z. noch 1,42 gr vom Körper ab, setzte am 2. und 3. 0,66 und 0,96 an, d. h. in den 3 Tagen im ganzen $0,20 \mathrm{gr}$, während er bei Milcbdiät $9,37^{*} \mathrm{gr} \mathrm{N}$ eingebtisst hatte.

Stickstoffbilanz.

\begin{tabular}{c|c|c|c|c|c}
\hline & Einnahme & \multicolumn{3}{|c|}{ Ausgabe } & Am Körper \\
& & Harn & Koth & Summa & \\
\hline 1. & 17,37 & 17,30 & 1,49 & 18,79 & $-1,42$ \\
2. & 17,37 & 15,29 & 1,49 & 16,91 & $+0,66$ \\
3. & 17,37 & 14,92 & 1,49 & 16,41 & $+0,96$ \\
\hline Summa & 52,11 & 47,44 & 4,47 & 51,91 & $+0,20$
\end{tabular}

Vergleicht man mit dieser Stickstoffbilanz die der ersten Reihe, so findet sich eine erheblicho Sparwirkung der zugegebenen Kohlenhydrate und des Fettes. Dort waren bei Aufnahme fast der gleichen Menge Stickstoff $(49,3 \mathrm{gr}$ gegen 52,1$) 9,6 \mathrm{gr} \mathrm{N}$ vom Körper abgegeben, hier $0,2 \mathrm{gr}$ angesetzt. $\frac{9,8.100}{52}=171 / 2 \%$. Voit (9) griebt den Sparwerth der Fette und der Kohlenhydrate zu höchstens $15 \%$ an. Vergleicht man unter Weglassung je des ersten Versuchstages die Ergebnisse je der zwei letzteren mit eiuander, so ist das Resultat ein noch guinstigeres:

\begin{tabular}{l|c|c|c|c} 
& Aufnahme & Ansatz & Aufnohme & Ansatz \\
\hline 2. Tag & 17,12 & $-3,20$ & 17,37 & $+0,66$ \\
$3 ., \%$ & 17,65 & $-3,14$ & 17,37 & $+0,96$ \\
\hline Summa & 34,77 & $-6,34$ & 34,74 & $+1,62$
\end{tabular}

Gespart in Reihe B 6,34+1,62=7,96 gr N $=23 \%$. Die Kürze der Versuchsreihen giebt wohl kanm Anlass zu Einwänden, um so weniger, als ja in Reihe A das Stickstoffdefizit keine Tendenz zurVerringerung zeigte, wohl aber in Reihe B der Ansatz von Tag zu Tage zunahm. Vielleicht wiirden grade längere Reihen eine noch erheblichere Sparwirkung zeigen. 
Bekannt ist, dass bei ausschliesslicher Milchkost die Fäulniss im Darm minimal ist. Der Koth ist fast geruchlos und die Menge der gebundenen Schwefelsäure im Harn stark vermindert. Ihre Menge beträgt gewöhnlich $1 / 9-1 / 12$ der präformirten Schwefelsäure, fällt auf $1 / 30-1 / 40$ bei Milchkost. Die Mengen der präformirten und der gebundenen Schwefelsäure (als $\mathrm{SO}_{3}$ berechnet) wurden je an 3. Tage beider Vesuche bestimmt, ausserdem später die gleichen Bestimmungen an einem während dreier Tage bei beliebiger Kost aufgefangenen Urin gemacht.

\begin{tabular}{|c|c|c|c|c|c|c|}
\hline & $\begin{array}{c}\mathrm{A} \\
\text { Präform. } \\
\mathrm{SO}_{3}\end{array}$ & $\begin{array}{c}\mathrm{B} \\
\text { Gebun- } \\
\text { dene } \mathrm{SO}_{3}\end{array}$ & $\begin{array}{c}\text { Ge- } \\
\text { sammte } \\
\mathrm{SO}_{3}\end{array}$ & $\frac{\mathrm{A}}{\mathrm{B}}$ & $\begin{array}{l}\mathrm{N} \text { im } \\
\text { Harn }\end{array}$ & $\begin{array}{l}\text { VerhäItniss } \\
\text { des } \mathrm{N} \text { zum } \\
\left.\mathrm{S} \text { in } \mathrm{d} . \mathrm{SO}_{3}{ }^{1}\right)\end{array}$ \\
\hline 3. Milchtag & 2,799 & 0,081 & 2,880 & 35 & 19,94 & 17,3 \\
\hline 3. Brodmilchtag & 2,168 & 0,054 & 2,222 & 40 & 14,92 & 16,8 \\
\hline Frei gew. Kost & 2,004 & 0,131 & 2,135 & 15,2 & 12,83 & 15,0 \\
\hline
\end{tabular}

Wie aus der kleinen Tabelle bervorgeht, war somit auch bei einer Nahrung, die etwa $1 / 3$ der Spannkraft in Form von Milch zufuhhrte, die ausser dem Milchcasein allerdings nur pflanzliches Eiweiss und viel Amylaceen enthielt, die Darmfäulniss ebenso stark gebemmt, wie bei reiner Milchdiät. Das steht durchaus in Uebereinstimmung mit den Erfahrungen von Fr. Müll er (10), Ortweiler (11), Hirschler (12), Hoppe-Seyler (13), Poehl (14), die die Darmfäulniss bei vorwiegendem oder ausschliesslichem Genuss von Pflanzeneiweiss, Amylaceen, Milch wie bei reinem Vegetarianismus sehr beschränkt sahen, während $B$ i e r n a ck i (15) - nicht mit Recht - jene fäulnisshemmende Wirkung nur der Milch zuerkennen will.

Die Ausnutzung der Nahrungsmittel, ausgedriiekt in Verlustprocenten der Bestandtheile, hatte sich in den beiden Versuchen demnach gestellt $\mathrm{zu}$ :

\begin{tabular}{|c|c|c|c|c|c|c|}
\hline & $\mathbf{N}$ & "Fett ${ }^{4}$ & Kohlenh. & Asche & Tr. S. & Org. Subst. \\
\hline Milch & 5,1 & 4,5 & - & 29,2 & 5,5 & $4 \%$ \\
\hline Brod & 13,7 & - & -1 & 8 & 1001 & 12701 \\
\hline Butter & - & 8,7 & $-\}$ & 28,9 & $4,0 \%$ & \\
\hline
\end{tabular}


Beiträge zur Kenntniss der Verdaulichkeit der Milch und des Brodes. 553

Frthere Untersucher haben für Kuhmilch folgende Verlustzahlen gefunden:

\begin{tabular}{|c|c|c|c|c|c|c|}
\hline & $\left|\begin{array}{c}\text { Menge d. } \\
\text { am Tage } \\
\text { genossen. } \\
\text { Milch } \\
\text { in Litern }\end{array}\right|$ & 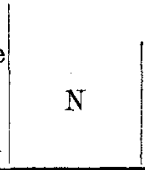 & „Fett“ & Asche & Tr. S. & $\begin{array}{l}\text { Org. } \\
\text { Subst. }\end{array}$ \\
\hline Prausnitz (3) 1 Erwachs. 3 Tage & 3,0 & 11,2 & 5,1 & 37,1 & 9,0 & 6,95 \\
\hline 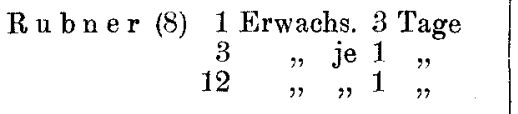 & $\begin{array}{c}2,44 \\
2,1-4,1\end{array}$ & $\left\{\begin{array}{l}7,0 \\
-\end{array}\right.$ & $\begin{array}{l}4,5 \\
-\end{array}$ & ea. 47,0 & $\begin{array}{l}8,5 \\
-\end{array}$ & $\begin{array}{c}6,4 \\
5-6\end{array}$ \\
\hline Uffelmann (16) 1 Erw. $3 \times$ je 1 Tag & $1,5-1,8$ & - & 5,8 & 48,0 & 8,2 & 6,5 \\
\hline $\begin{array}{c}\text { Sassetzky (17) ca. } 10 \text { Erw. je ? } \\
\text { (Reconvalescenten) }\end{array}$ & - & $\frac{4,0-8,1}{6,7}$ & - & - & - & - \\
\hline $\begin{array}{c}\text { Hoesslin (18) } 4 \text { Erwachs. je ? Tage } \\
\text { (Typhuskranke) }\end{array}$ & ? & $2,2(?)-10,0$ & $6,4-10,8$ & - & - & - \\
\hline Hoffmann (4) 1 Erw. $2 \times 3$ Tage & ca. 2,8 & $11 \%$ & - & - & - & - \\
\hline $\begin{array}{c}\text { Laptschinsky }{ }^{1} \text { ) (19) } 5 \text { Erwachsene } \\
\text { je } 5-7 \text { Tage }\end{array}$ & $2,4-3,4$ & $6,7 \%$ & - & - & - & - \\
\hline $\begin{array}{c}\text { Slatk owsky (7) } 3 \text { Erwachs., } 7 \text { Ver- } \\
\text { suche à } 5 \text { Tage }\end{array}$ & $3,0-5,2(!)$ & $5 \%$ & - & - & 一 & - \\
\hline Herfeldt (5) 1 Erwachs. 2 Tage & 3,0 & 5,1 & 6,9 & 39,4 & 6,1 & 4,2 \\
\hline Fr. Müller (20) 1 Erwachs. 2 Tage & $2,2-2,5$ & 5,9 & 7,2 & 38,2 & 8.1 & 6,0 \\
\hline $\begin{array}{l}\text { Klemperer (25) } 2 \text { Erwachsene } \\
\text { Inanitionsstadium je } 3 \text { Tage }\end{array}$ & 1,5 & 8,0 & - & - & ca. 17,7 ! & 一 \\
\hline $\begin{array}{c}\text { Camerer (6) } 5 \text { Kinder je } 4 \text { Tage } \\
(4-12 \text { Jahre })\end{array}$ & $1,7-2,0$ & $\underbrace{3}_{6,3(?)-9,3}$ & $\underbrace{2,8-6,1}_{1}$ & $\underbrace{47,0-48,0}$ & $\frac{4,4-7,1}{9}$ & 4,5 \\
\hline,$\quad 1$ Kind mehr. Tage (7 Mon.) & $?$ & $\begin{array}{l}1,3 \\
8,2\end{array}$ & $\underline{4,6}$ & $-4,6$ & ca. 6,3 & - \\
\hline Forster (21) 1 Kind 11 Tage (4 Mon.) & 1,0 & - & - & - & 6,4 & 4,5 \\
\hline Uffelmann(16) 4 Säuglinge 2-7 Tage & $0,6-2,1$ & - & 6,2 & 48,3 & 7,5 & 5,5 \\
\hline $\begin{array}{c}\text { Magnus-Levy (16 jähr. junger } \\
\text { Mann) } 3 \text { Tage }\end{array}$ & 3,5 & 5,1 & 4,5 & 29,2 & 5,5 & 4,0 \\
\hline
\end{tabular}

1) In den Versuchen von L., wurde neben Milch noch "Gebäck" gegeben, 
Die Verwerthung der organischen Substanz und des Stickstoffs war also etwas besser als in den meisten Versuchen deutscher Autoren, speciell an Erwachsenen, und erreichte die Ausnutzung bei Kindern, die bekanntlich die durch Erwachsene hinter sich. lässt. Die Resorption des Fettes wich nicht sehr von der in anderen Versuchen beobachteten ab. Auffallend erscheint nur die relativ starke Aufsaugung der Aschenbestandtheile (29\% Verlust gegen $37-48 \%$ bei Rubner u. a.), da der gut ernährte Organismus doch kaum ein Bedürfniss nach denselben haben konnte. Für den Haushalt des Erwachsenen spielt dies aber wohl keine Rolle.

Ausserordentlich gut wurde der Stickstoff des Brodes verwerthet, da nach obiger Annahme nur $13,7 \%$ desselben zu Verlust gingen. M e y e r (22) und $R$ u bner (8) sahen in ihren besten Versuchen 18,7 und $19,9 \% \%^{\mathrm{j}}$ ) Verlust an Stickstoff, während $\mathrm{Z}$ u n tz und des Verfassers Versuche mit 15,5 und $16,1 \%$ Verlust $\mathrm{N}$ von den Resultaten des vorliegenden. Experiments nicht sehr stark abweichen. Die Verwerthung der organ. Substanz des Brodes geschah in unserem Versuch bis auf einen Rest von 3,7\%, Rubner giebt diesen ähnlich zu 3,7 und $5,2 \%$ an; $\mathrm{Zuntz}$ und MagnusLevy fanden ihn in ihren guten Brod-Butter-Versuchen zu $2 \frac{1}{2} \%$ (L.) und $3 \%$ (Z.). Ein Verlust des Butterfettes von 8,7\% im Koth übersteigt wesentlich die von $\mathrm{R} \mathrm{ubner}$ und dem Verfasser unter ähnlichen Bedingungen gefundenen Zahlen 1,8-3,7\% (R.) 2,3 bis $5,4 \%$ (Z: u. M.-L.) (selbst bei Diarrhoen).

Lässt sich aus diesen Versuchen ein Beweis für die Annahme Bunges herleiten, dass Analcoholiker die Nährstoffe besser ausnutzen, als solche, die mässige Mengen Alcohol geniessen? Verfasser glaubt diese Frage nicht unbedingt bejahen zu müssen. Allerdings stellte sich die Verwerthung der organischen Sub-

entsprechend 1,7-4,0 gr N. Die Ausnutzung des Stickstoffs in der Milch war daher noch günstiger als die betreffende Zahl angiebt, die den $\%$-Gesammtverlust an Stickstoff bedeutet. Dagegen ist aus oben angeführten Gründen bei $\mathrm{Ru} b$ ne $\mathrm{r}$ die Ausnutzung des Stickstoffs vielleicht noch ungünstiger gewesen, als er selber sie berechnet.

1) Es wäre interessant zu wissen, ob die Individuen, an denen die russischen Forscher ihre Versuche anstellten, die die grössten Milchmengen vertrugen und wenigstens den Stickstoff so gut ausnutzten, an regelmässigen Alcoholgenuss gewöhnt waren oder nicht. 
Beiträge zur Kenntniss der Vordaulichkeit der Milch und des Brodes. 555 stanzen der Milch um 2-3\% besser als in den Versuchen von Praussnitz, Rub ner und Hoesslin; ferner konnte man zu Gunsten des Baseler Physiologen anführen, dass Verfasser seine Absicht, mit Alcoholgenuss (Cognac) einen Parallelversuch an sich anzustellen, wegen heftiger Beschwerden nicht durchführen konnte. Nun nimmt aber die Miich eine Sonderstellung unter den Nahrungsmitteln ein: ausschliesslich, oder auch nur in grosseu Mengen genossen, verursacht sie zahlreichen Menschen heftige Leibschmerzen, Durchfälle and nervöse Beschwerden, die ihre geeignete Verwerthung erschweren oder unmöglich machen. Ob es der Alcohol ist, der bei den meisten Erwachsenen die Neigung und die Fähigkeit der Milchaufnahme verringert, wagt Verfasser nicht zu entscheiden; neben der fast völligen Abstinenz kommt jedoch für das günstige Ergebniss des Versuches bei dem jungen $Z$. die systematische Erziehung zum Milchtrinken in Betracht; der Verdanungsapparat eines Menscben, der täglich 11/2 Liter Milch zu verarbeiten hat, ist zur Aufnahme grösserer Mengen dieses Nahrungsmittels jedenfalls besser vorbereitet, als der eines Mannes, dem Milch als Getränk für sich kaum mehr bekannt ist.

$\mathrm{B}$ u n g' e's Finwande gegen die Versuche von $M$ e yer, $\mathbf{R}$ u bn e r u. a. sind gemacht anlässlich einer Zusammenstellung der Ausnutzung des Eiweisses in den verschicdener Nahrangsmitteln, und kann er sich daher zunächst nur auf das Eiweiss beziehen. Im ersten Augenblick scheint das Resultat unseres Versuches B u n ge recht za geben: $13,7 \%$ Verlust an N. gegen 18,7, $19,9^{\circ} / 0$ und 25,5 zeigen immerhin eine nicht zu unterschätzende Differenz, die aber durch Berücksichtigung folgende: Momente an Gewicht verliert. Zunächst scheint eine, wenn auch nur aus wenigen einfachen Bestandtheilen gemischte Nahrung (hier Brod, Butter, Milch) besser ausgenutzt zu werden, als eine ungemischte ${ }^{1}$ ). Weiter-

1) Sowohl Rubner wie auch Malfatti (23) (Wiener Akad. Sitzungsberichte 1884. Dee. Bd. 903) fanden die Ausnutzung je eines Nahrungsmittels (Mileh resp. Polenta) durch Zugabe von Käse ganz auffallend gebessert. Selbst unter Annahme, dass der Käse quantitativ resorbirt worden wäre, war die Ausnutzung noch wesentlich besser geworden. (I)m gegenüber sah M a lfatti allerdings Butter in zwei Versuchen die Resorption des Stickstoffs aus Mais and Erbsen nicht unerheblich verschlechtern.) - Auch die Versuche von Laptschinsky (19) sprechen zu Gunsten dieser Annahme. In 3 seiner Versuche war die Gesammtausnutzung des im Brod (ca. 1,7 bis 
hin kommen zweifelsohne individuelle Schwankungen in der Ausnutzung vor, wie solche u. a. durch des Verfassers citirte Versuche nachgewiesen werden konnte. Sie nutzten in ihren (gelungenen) Selbstversuchen den N. des Brodes zu 16,1 und 15,5\% aus; die niedrigere Zahl von $15,5 \%$ entfernt sich nicht zu weit von der entsprechènden in unserem Versuch von $13,7 \%$; sie wurde gefunden bei einem täglichen Consum von 1,8 Liter Bier, der für L. das Mittelmaass seines Alcoholverbrauchs darstellt.

Einer vielleicht etwas besseren Verwerthung des Stickstoffes steht in unserem Versuch ein grösserer Verlust an Fett gegenüber. Auch in Z. und M.-L's. Doppelexperimenten batte der Alcoholconsument etwas weniger Fett ungenïtzt abgegeben $(2,3$ und $4,5 \%$ ) als der Abstinenzler (5,4 und 4,8\%).

Ohne dieser Thatsache besonders grossen Werth beilegen zu wollen, kann man trotzdem daran erinnern, dass reichlicher Fettgenuss, besonders bè copiösen Mahlzeiten, viele Menschen veranlasst, Alcohol, in seiner concentrirtesten Form, als Branntwein (Cognac u. a.) zu sich nehmen; er hat hier vielleicht ausser der subjectiven Wirkung auch eine objective zu erfüllen, so dass die Geschmacksrichtung für eine Ueberladung nach der einen Seite sich ein - freilich durchaus nicht einwandfreies Corrigens sichert.

Berücksichtigt man nun bei der Ausnuitzung der Nahrungsstoffe im Darm des Alcoholikers und des Abstinenzlers nicht die Ausnutzung des Eiweisses und Fettes allein, sondern die der gesammten organischen Substanz, so verschwinden etwaige Unterschiede fast ganz, da ja die Kohlenhydrate, die uns den grössten Theil der Nährstoffe und der Spannkraft zufiuhren, fast stets gänzlich resorbirt werden, wenn man sie nicht in ungeeigneter Form und Menge zuführt. $R \mathfrak{u} b \mathrm{n}$ er fand die organische Substanz des Weissbrodes zu 96\% ausgenutzt, die entsprechende Zahl für Weissbrod und Butter in unserem Versuche ist $96,3 \%$ resp. 97,1, bei Beziehung des fettfreien organischen Kothes (nach Abzug des auf die Milch treffenden Theiles) auf das Brod allein. Prof. Zuntz hatte ca. $97 \%$, der Verfasser (bei Biergenuss) $97,5 \%$ aufgesangt.

4,0 gr N) und Milch enthaltenen Stickstoffes glänzend (Verlust $=3,9,5,0$ und $5,7 \%$ d. h. noch besser als sie sich mit Zugrundelegung der in unserem Versuch gewonnenen Zahlen ergeben würde. 
Beiträge zur Kenntniss der Verdaulichkeit der Milch und des Brodes. 557

Das sind Unterschiede von solcher Kleinheit, dass sie für den Gesammthausbalt verschwinden und in das Bereich individueller Schwankungen fallen.

Es scheint somit keine für den Gesammtumsatz wesentlich in Betracht kommende Schädigung der Ausnutzung der menschlichen Nahrung durch mässigen dauernden Alcoholgennss zu Stande zu kommen. Keine Verschlechterung der Gesammt-Ausnutzun. wohlverstanden, wie sie sich in ad hoe angestellten Versuchen ergibt, womit über die Bedentung und sonstige Sebädlichkeit des Alcoho s im Ganzen nichts bewiesen ist. Der Schaden, den der Spiritus vini et cerevisiae stiftet, liegt jedenfalls nicht in dieser Riehtung. In Betracht kämo höchstens eine etwas bessere Ausuutzung des Eiweisses, die ja freilich nicht zu unterschätzen ist, da bei dem chronischen Stickstoffimangel in der Nahrung weiter Kreise ein Plus oder Minus von 5-10 gr. Liweiss zweifellos eine grössere Bedeutung hat, wie etwa ein solehes von der gleichen Menge Stïke. $W(n n$ aber Verfasser bei einem von $B$ unge bereits beanstandeten Bierconsum von $1{ }^{1} / 2-2$ Liter Bier fast ebenso günstige Resultate errielte, wie der Analcoholist dieses Versuches, und bessere als friihere Experimentatoren, so kam man doch eventuell noch eine andere Erklärung dafï suchen. Wer unter giirstigen Lebensund Ernährungsbedingungen steht, besitat jedenfals -- normale Lebensfülurung voransgesetzt -- neben einem kräftigen Muskelapparat auch ein besser functionirendes Verdaunngssystem. Reichere Liweisszufuh - and dicse ebaracterisirt in erster heihe die hessere Speise der Wohlhabenden - scheint cine bessere Secretion und Resorption hervorzurufen, und sowit auch eine bessere Verwerthung des zeitweise in geringerer Menge zugefïhrten Eiweisses za bedingen.

Bei R ub er und M c y er dienten zu den Versuchen Leute, deren Lebenshaltung eine geringere war, als in den unserigen ${ }^{1}$ ). Sollte bei Alcoholgenuss wirklich - abgesehen von sonstigen hier nicht in Frage kommenden schädlichkeiten - cine schlechtere Ausnutzung der Nahrung statthaben, so ist doch der Alcohol nicht die alleinige und directe Ursache dieser Versehiechterung der materiellen Lebensfiihrung.

1) In Rub ner's Versuchen der Laboratoriumsdiener; M e y e r giebt, allerdings an, dass sein Individum sich guter Verdaung orfrente und Brod gewöhnt will. 
Einen gewissen Auhalt für diese Anschauung finden wir in J. M unk's Versuch am Hund ${ }^{1}$ ). Als in einer ausreichenden Nahrung dieses Hundes ein grosser Theil des Eiweisses durch mehr als äquivalente Mengen von Stärke ersetzt wurden, fing nach einer Reibe von Wochen der Hund an schlecht zu resorbieren; die Eiweissaufsaugung wurde immer schlechter. Der chronische Stickstoffhunger so vieler Menschen mag anch bei ihnen eine schlechtere Secretion und Resorption bedingen, wenngleich auch das Deficit, relativ viel geringer als im M un k'schen Versuch, es nicht zu so erheblichen Störungen kommen lässt, wie sie in jenem Versuch auftraten.

Mit einigen Worten sei hier noch eingegangen auf die Frage: Wird die "Verdaulichkeit" der Milch durch Kochen resp. Sterilisiren derselben wesentlich verändert? $R$ a u d n itz (24) findet eine nicht unerhebliche Verschlechterung der Sticktoffverwerthung bei sorgfältigen Versuchen, die er am Hund zur Lösung dieser Frage angestellt hat. In unserem Fall war die sterilisirte Milch so gut verwerthet worden, wie in den besten vorliegenden Mittheilungen, d. h. so gut wie sie iiberhaupt nur verwerthet werden konnte. Angaben über die. Zubereitung der Milch finden sich nur bei $\mathrm{Camerer}$, der seine Kinder die Milch gekocht geniessen liess, bei $\mathrm{R} u \mathrm{~b} n \mathrm{er}$, der sie theils roh, theils gekocht gab, und bei Prausnitz, der die Mileh in Literflaschen zwei Stunden im K o c h'schen Dampftopf bei strömendem Dampf sterilisirte. Der Unterschied zwischen den Resultaten des Verfassers und denen von $\mathrm{R} \mathrm{a} \mathrm{udnitz}$ sind wahrscheinlich dadurch zu erklären, dass letzterer seine Milch im Dampftopf 1 Stunde bei $1 / 2$ Atmosphäre Ueberdruck, d. h. bei $112^{\circ}$ erhitzte, wodurch sie "leicht gebräunt" wurde. Dagegen ist die zum Vertrieb im Grossen ebenfalls in Literflaschen hergestellte Milch laut gefälliger Mittheilung der Meierei Bolle mit strömendem Dampf bei nur $102^{\circ}$ behandelt. Diese Sterilisation ist eine so vollkommene, dass die Milch nach monatelangem Stehen in den verschlossenen Flaschen keine „Zersetzung" zeigte (abgeseben natiurlich von Aufrahmung). Bis also

1) Eine vorläufige Mittheilung findet sich in den Berichten der Berliner physiol. Gesellsch. (D u bo is' A rchiv 1891 S.388. Spätere Versuche dieser Autoren bestätigein nach einer mündlichen Mittheilung dieses Autors die Resultate der dort migetheilten Versuchsreibe. 
Beiträge zur Kenntniss der Verdaulichkeit der Milch und des Brodes. 559

neue gegentheilige Erfahrungen vorliegen, darf man annehmen, dass die gewerbsmässig betriebene Sterilisation der Milch, die berechtigten Anforderungen durchaus erfüllt, die Verwerthung derselben nicht schädigt.

Herrn Prof. $Z$ u $n$ tz bin ich auch für diese Arbeit dankbar verpflichtet; er hat mir das Thema, seinen Rath und last not least seinen Sohn zur Verfügung gestellt. Diesem selber, Herm Leo $\mathrm{Z}$ a n t $\mathrm{z}$, habe ich aufrichtigen Dank auszusprechen, für das liebenswiurdige Entgegenkommen und die Selbstverläugnung, mit der er sich diesen Versuchen unterzogen hat.

\section{Litteratur.}

1) G. Bunge: Lehrbuch d. physiol, u. patholog. Chemie, 2. Aufl. Leipzig 1889. S. 72.

2) Zuntz u. Nagnus-Levy: Beiträge z. Kenntniss der Verdaulichkeit ete. des Brodes. Pfüger's Archiv Bd. 49. S. 438.

3) Prausnitz: Ueber die Ausnutzung d. Kuhmileh im menschl. Darmcanal. Ztschr. f. Biologie Bd. 25. S.53i\%.

4) Hoffrnann: Betrachtungen über absolute Milchdiät. Ztschr. f. klin. Medicin. Bd. 7. Anhang S. S.

5) II erfeldt: Mittheilungen aus d. med. Klinik zn Würzburg. Bd. I. S. 59; ausfilhrl Angabe bei Müller: Icterus.

(i) Camerer: Stoffwechsel eines Kindes. Ztschr. f. Biologie. Bd. 14, S. 383. - Stoffwechsel von Kindern bei ausschliessl. Ernährung mit Kuhmilch. Z7tschr. f. Biologie Bd. 16. s. 493. Bd. 18. S. 488.

7) Slatkowsky: Ausführl. Angaben bei Hoffmann.

s) Rubner: Ueber die Ausautzung ete. Ztsehr. f. Biologie. Bd. 15. S. $130 f f$.

9) Voit: Physiologie des allgemeinen Stoffwechsels. S. 130 u. 140.

10) l'r. Mäller: Indicanausscheidung durch den Darm. Mittheilungen aus der Würzburger med. Klinik. Bd. 2. S. 341.

11) Ortweiler: Bedentung des Hamindicans. Ibidem S. 153.

12) Hirschler: Ueber den Einfluss der Kohlenhydrate ete. auf d. Eiweissfäulniss. Ztschr. f. physiol. Chemie. Bd. X. S. 306.

13) Hoppe-Seyler: Ueber die Ausscheidung der Aetherschwefelsäure im Urin bei Krankheiten. Ibidem Bd. XII. S. 1.

14) Poeh 1: Uober Narmfäunniss. Maly's Jahresbericht 1887. Bd. 17. S. 277. 
15) Biernacki: Ueber die Ausscheidung der Aetherschwefelsäuren etc. Centralblatt für med. Wissenschaft. 1890. S. 881 u. 898; auch Deutsches A rrchiv für klin. Med. Bd. 49. S. 96.

16) Uffelmann: Studien über die Verdaulichkeit der Kuhmilch. Pflüger's Archiv Bd. 29. S. 339.

17) Sassetzk.y: Virchow's Archiv. Bd. 94. S. 506.

18) Hoesslin: Ernährung fiebernder Kranker. Virchow's Archiv. Bd. 89. S.95.

19) Laptschinsky: Citirt bei Hoffmann.

20) Fr. Müller: Untersuchungen über Icterus. Ztschr. für klin. Medicin. Bd. 12. S. $44 \mathrm{ff}$.

21) Forster: Aerztl. Intelligenzblatt für Bayern 1878. März.

22) Meyer: Ernährungsversuche mit Brod. Ztschr. für Biologie. Bd. 7. S. 1.

23) Malfatti: Wiener akad. Sitzungsberichte. 1884. December. Bd $90^{3}$.

24) Raudnitz: Ueber die Verdaulichkeit gekochter Milch. Ztschr. f. physiol. Chemie. Bd. 14. S. 1 .

25) Klemperer: Stoffwechsel und Eruährung in Krankheiten. Ztschr. f. klin. Medicin Bd. 16. Heft 5 u. 6.

(Aus dem Laboratorium für experimentelle Pathologie des Herrn Professor Dr. S. v. Basch in Wien.)

\section{Ueber die Innervation der Blase.}

Von

\section{Dr. Maximilian von Zeissl,}

Privatdocent in Wien.

Mit 8 Holzschnitten

Die Frage, wie der Verschluss der Harnblase zu Stande komme, beschäftigte die Aerzte schon seit langer Zeit. Dieselbe hat aber bisher trotz vieler darauf abzielender Versuche noch keine endgiltige Entscheidung gefunden. Hieran sind sowohl die complicirten anatomischen Verhältnisse als auch die Methoden, nach welchen bisher experimentirt wurde schuld. Da B or ${ }^{1}$ )

1) Zur Kritik über den gegenwärtigen Stand von der Frage von den Blısenfunctionen. Deutsche Zeitschrift für Chirurgie, redigirt von $\mathrm{L}$ ü $\mathrm{cke}$ und $\mathrm{R}$ os e. Band 25, 1887, Seite 118-192. 